\title{
Evaluación del programa "Active Communication Education" para rehabilitación auditiva en adultos mayores con hipoacusia usuarios de audífonos
}

\section{Assessment of the "Active Communication Education" program for audiological rehabilitation in patients with hearing loss users of hearing aids}

\author{
Felipe Cardemil ${ }^{1,2}$, Tamara Barría ${ }^{2}$, Lorena Aguayo², Patricia Esquivel'1, Maritza Rahal', \\ Adrián Fuente ${ }^{3}$, Rocío Carvajal ${ }^{3}$, Rose Marie Fromin ${ }^{3}$, Iván Villalobos ${ }^{4}$.
}

\begin{abstract}
RESUMEN
Introducción: La hipoacusia es una condición prevalente en adultos mayores. Sin embargo, la baja adherencia al uso del audífono representa una realidad.

Objetivo: Evaluar la utilidad de un programa de intervención para mejorar adherencia en pacientes con hipoacusia mayores de 65 años usuarios de audífono.

Material y método: Ensayo clínico aprobado por Comité de Ética. Se incluyeron pacientes beneficiarios del GES de hipoacusia. Se evaluó adherencia al audífono en un corte a 6 meses.

Resultados: Fueron evaluados 180 pacientes, correspondiendo a 90 pacientes control y 90 intervenidos. La adherencia global al uso del audífono fue de $78 \%$. No hubo diferencia en los grupos por sexo, edad, ni grado de hipoacusia. El promedio de horas diarias de uso en el grupo control fue de 6,9 horas, comparado con el del grupo intervenido que fue 9,6 horas $(p<0,0001)$. Hubo una asociación significativa entre la intervención y la regularidad del uso del audífono, con $80 \%$ de pacientes que lo usaban regularmente en el grupo intervenido contra el $8,9 \%$ en el grupo control $(p<0,001)$.

Discusión: La intervención implementada ha demostrado excelentes resultados preliminares en términos de tiempo de uso del audífono, por lo que sería de utilidad implementarla como parte de la política GES y difundir su uso.
\end{abstract}

Palabras clave: Hipoacusia, programas de rehabilitación, adherencia.

\footnotetext{
1 Médico, Servicio de Otorrinolaringología, Hospital Barros Luco-Trudeau, Facultad de Medicina, Universidad de Chile.

2 Médico, Escuela de Salud Pública, Universidad de Chile.

3 Fonoaudiólogo, Escuela de Fonoaudiología, Universidad de Chile.

4 Fonoaudiólogo, Servicio de Otorrinolaringología, Hospital Barros Luco Trudeau.
} 


\section{ABSTRACT}

Introduction: Hearing loss is a prevalent condition in elderly population. However, the low adherence to hearing aids is a fact.

Aim: To evaluate the utility of a standardized counselling program in elderly patients with hearing loss.

Material and methods: A clinical trial was carried out, approved by the Hospital ethics committee. Patients with 65 years and older with hearing loss were included. We assessed adherence at a follow-up of 6 months.

Results: 180 patients were evaluated, corresponding to 90 control and 90 rehabilitated patients. The overall adherence of hearing aids use was $78 \%$. There was no difference in sex, age, and level of hearing loss between groups. The average daily hours of use in the control group was 6.9 hours, compared with the 9.6 hours in the intervention group $(p<0.0001)$. There was a significant association between the intervention and regular hearing aid use, with $80 \%$ of patients who used it regularly in the intervention group compared with $8.9 \%$ in the control group $(p<0.001)$.

Discussion: The intervention implemented has shown excellent preliminary results in terms of time of use of the hearing aid. It would be useful to include this intervention in the hearing loss national public policy.

Key words: Hearing loss, rehabilitation programs, adherence.

\section{INTRODUCCIÓN}

La hipoacusia es una condición muy prevalente, sobre todo a mayor edad de las personas ${ }^{1,2}$. La pérdida de audición de 25 dBo más afecta a alrededor del 37\% de los adultos de 61 a 70 años de edad, $60 \%$ de los adultos de 71 a 80 años de edad, y más del $80 \%$ de los adultos mayores de 85 años ${ }^{3,4}$. De acuerdo a cifras de la Organización Panamericana de Salud (OPS), esta cifra fluctúa entre el $30 \%$ en mayores de 65 años hasta $60 \%$ en mayores de 85 años $^{5}$. En adultos mayores chilenos, la prevalencia se estima en $77,8 \%$, cifra que se eleva a $91,1 \%$ en mayores de 80 años, de acuerdo a datos de la Encuesta Nacional de Salud $2003^{6}$.

Se ha asociado a la hipoacusia con disminución en la calidad de vida y trastornos psicológicos ${ }^{1,7}$. A menudo, es subestimada por ser un problema de lenta progresión o debido a la creencia de que la pérdida de audición es una parte normal de envejecimiento. La hipoacusia afecta la calidad de vida de quienes la padecen, en particular el funcionamiento psicológico, social y emocional ${ }^{8}$. En términos sociales puede traer varias consecuencias en las relaciones interpersonales y en las actividades grupales ${ }^{9}$. Las variables psicológicas que se han asociado con hipoacusia son múltiples: depresión, soledad, ansiedad, somatización y funcionamiento social pobre ${ }^{8}$, siendo la depresión la enfermedad psiquiátrica más frecuentemente relacionada con hipoacusia en adultos ${ }^{10}$. Esta asociación no es causal, pero se podría plantear que la hipoacusia sin tratamiento tendría un impacto en la salud mental de quienes la padecen.

Desde julio del año 2007, se incluye dentro de las Garantías Explícitas en Salud (GES) la entrega de audífonos a personas de 65 años y más con hipoacusia bilateral que lo requieran ${ }^{11}$. Esto, porque existe información que apoya el que la hipoacusia genera costos importantes para un país, debido a disminución de la productividad, educación especial, y atención en salud ${ }^{12}$. La Guía Clínica GES establece como parte del flujograma de seguimiento la realización de programas de consejería en rehabilitación audiológica, y de valoración y calibración del audífono en caso de ser necesario ${ }^{11}$, no estableciendo un lineamiento claro respecto de qué modalidad de rehabilitación seguir. Sin embargo, no existen estudios en Chile que demuestren efectividad de alguna de las dos intervenciones ${ }^{13}$. Además de esto, hay que considerar que la entrega de audífonos va aumentando exponencialmente, estimándose que en Estados Unidos ha experimentado un incremento de $300 \%$ en el período $1996-2006^{14}$.

Dentro de los problemas prácticos en el uso de audífonos se encuentra la inserción, eliminación 
y limpieza del dispositivo, el cambio de pilas, el control del volumen, y los ruidos "extraños"15. En cuanto a la adherencia a su uso, a pesar de muchos beneficios bien documentados, sólo el $25 \%$ de los pacientes elegibles adquiere audífonos y hasta el $30 \%$ a $50 \%$ de éstos no los utilizan ${ }^{1,16,17}$. En nuestro país, la cifra aceptada de adherencia se sitúa en torno a $53 \%{ }^{3}$, pero existen reportes que varían entre el $40 \%$ y $80 \% 5,6$. Al revisar los datos reportados respecto a los factores asociados a la baja adherencia, se menciona el efecto negativo del ruido ambiental, la poca comodidad, la falta de motivación, dudas sobre funcionamiento, poca accesibilidad a servicio técnico, la pobre apariencia cosmética y mala disposición previa, entre otros ${ }^{1,17-19}$. Algunos autores mencionan que sólo 1 de cada 8 adultos con indicación de audífonos acepta su uso y que sólo el $85 \%$ de los pacientes usaría correctamente éste ${ }^{20,21}$. Es importante el asesoramiento de los pacientes con pérdida de audición, porque las percepciones y expectativas de ellos se mencionan como factores muy relevantes en la adquisición de audífonos y su adherencia ${ }^{21-24}$.

\section{OBJETIVO}

El objetivo del presente estudio fue evaluar la utilidad de un protocolo de consejería estandarizado basado en el programa "Active Communication Education" (ACE) ${ }^{25}$ como intervención en pacientes con hipoacusia usuarios de audífonos para mejorar la adherencia a su uso.

\section{MATERIAL Y MÉTODO}

Se realizó un ensayo clínico antes-después para el que se comparó un grupo de pacientes que había recibido el audífono y fue expuesto a la intervención (rama activa) con un grupo de pacientes que había recibido su audífono previamente y que no fueron sometidos a la intervención (rama pasiva), durante el período octubre 2011 a agosto de 2012, en un centro hospitalario docente. Se incluyeron pacientes beneficiarios del programa GES de hipoacusia. El protocolo contó con aprobación del Comité de Ética del Servicio de Salud Metropolitano Sur, y con firma del consentimiento informado de cada paciente. El protocolo fue inscrito en el registro en ensayos clínicos ClinicalTrials.gov (NCT01846676).
La intervención consistió en la aplicación de un programa de rehabilitación auditiva modular semiestructurado basado en el programa ACE. Este programa está enfocado para personas con pérdida de audición y se basa en estrategias de solución de problemas ${ }^{25}$. En su versión original, consiste de una serie de cinco módulos en grupos de 5 pacientes con sus familiares, acerca de actividades de la comunicación diaria, para las cuales la evidencia científica sugiere que son situaciones problemáticas para adultos mayores con hipoacusia y sus familiares cercanos, como por ejemplo la utilización del teléfono, escuchar la televisión, ir a un restaurante, o mantener una conversación durante la cena. Los módulos específicos que se abordan durante las sesiones del programa dependen de las necesidades comunicativas que son identificadas por el grupo de participantes durante la primera sesión. Este programa es por tanto menos prescriptivo que otros programas de comunicación, ya que los contenidos varían dependiendo de las dificultades de comunicación específicas descritas por los participantes. Actualmente el programa ACE se aplica en Australia, Nueva Zelanda, el Reino Unido y Escandinavia. Para el presente estudio, y de acuerdo a las guías GES, se adaptó el programa original a 4 sesiones en grupos de 4 personas, cada una con un acompañante. Cada sesión tuvo una duración de 1 hora y media, realizándose una vez por semana.

El seguimiento de los pacientes se realizó en un corte puntual a los 6 meses desde que les fue entregado el audífono. El desenlace primario a evaluar fue la adherencia al uso del audífono. La OMS define la adherencia terapéutica como la magnitud con que el paciente sigue las instrucciones médicas ${ }^{26}$, pero según Haynes y Sackett se define como la medida en que la conducta del paciente en relación con la intervención coinciden con las indicaciones dadas por su médico ${ }^{27}$, por lo que en esta ocasión se evalúo la adherencia aplicándose el International Outcome Inventory for Hearing Aids (IOI-HA) o Cuestionario Internacional del Resultado de Uso de Audífonos (CIRUA), considerando la primera pregunta de este instrumento, y un cuestionario de adherencia proporcionado por el Dr. Bevan Yueh (Anexo). Con éstos se estimó la adherencia en términos de uso general como variable dicotómica y con variables categóricas y continuas (Anexo). Además, se consideró la edad, 
Anexo. Cuestionario utilizado para evaluar adherencia al uso del audífono en pacientes con hipoacusia usuarios de audífono, basado en el cuestionario de adherencia proporcionado por Bevan Yueh, MD (preguntas 1 a 4), y el International Outcome Inventory for Hearing Aids (IOI-HA) (pregunta 5)

Cuestionario para evaluar programa Active Communication Education (ACE) para rehabilitación en pacientes con hipoacusia usuarios de audífonos

1. ¿Usted usa su audífono?

No

Sí

2. ¿Cuán a menudo usa su audífono?

Nunca

Sólo en raras ocasiones (vacaciones en familia, etc)

Ocasionalmente

Regularmente (cuando no estoy durmiendo o en la ducha)

3. ¿Cuántos días a la semana Ud. usa su audífono?

4. ¿Cuántas horas al día Ud. usa su audífono?

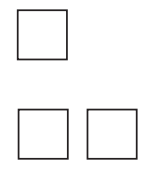

5. Piense cuánto ha utilizado usted su(s) audífono(s) actual(es) en las últimas dos semanas.

En un día común, ¿cuántas horas ha usado usted el(los) audífono(s)?

Ninguna

Menos de una hora al día

De 1 a 4 horas al día

De 4 a 8 horas al día

Más de 8 horas al día

sexo, nivel de pérdida auditiva considerando el promedio tonal puro, y el nivel de discriminación auditiva de los participantes.

Para el análisis estadístico se utilizó el software Stata11. Las variables continuas fueron descritas mediante promedio y desviación estándar o mediana y rango intercuartil (RIC) según correspondiera, y las variables categóricas mediante frecuencias absolutas y relativas. Se utilizó la prueba de Shapiro Wilk para evaluar la distribución de las variables continuas, y en base a si seguían una distribución normal o no, las variables continuas fueron comparadas con la prueba $t$ de student 0 el test de Mann Whitney, según correspondiera. Para las variables categóricas la comparación entre grupos se realizó utilizando el test exacto de Fisher. Se utilizó como nivel de significación un $\alpha=0,05$.

\section{RESULTADOS}

Se incluyeron 180 participantes, correspondiendo a 90 por rama. La adherencia global al uso del audífono fue de $78 \%$. El $33,89 \%$ fueron mujeres, sin haber diferencias significativas en cuanto a la distribución por género ni edad entre los dos grupos estudiados. No se apreció diferencia entre los grupos al evaluar edad $(p=0,32)$, sexo $(p=0,64)$, promedio tonal puro 
aéreo $(p=0,64)$ ni óseo $(p=0,56)$, ni discriminación auditiva $(p=0,57)$. De las 4 sesiones, el grupo de pacientes intervenidos presentó una asistencia promedio a $2,4( \pm 1,3)$ sesiones, con una mediana de 3 (RIC 1-3) sesiones.

Al evaluar el tiempo de uso se observó que en el grupo no intervenido el $42,2 \%$ usaba el audífono rara vez, y sólo el 8,89\% lo utiliza regularmente, mientras que en el grupo intervenido el $80 \%$ lo usa de manera regular y sólo 2,2\% refiere utilizarlo rara vez $(p<0,0001)$ (Tabla 1).

Respecto a la cantidad de días a la semana que los pacientes refieren usar sus audífonos, el grupo intervenido presenta un promedio de uso de 6,53 días $( \pm 1,1)$, y una mediana de 7 días $(R I C=2)$, comparado con un promedio de 6 días $(\underline{\underline{ \pm}} 1,7)$ y una mediana de 7 días $(\mathrm{RIC}=0$ ) en el grupo control, siendo esta diferencia significativa $(p=0,03)$ (Tabla 2).

Al evaluar el tiempo de uso al día de aquellos pacientes que lo utilizan regularmente mediante el cuestionario proporcionado por el Dr. Bevan Yueh (Anexo), el grupo no intervenido tuvo un promedio de uso de $6,9 \mathrm{hrs} /$ día $( \pm 3,55)$, mientras que el grupo intervenido lo usaba en promedio $9,6 \mathrm{hrs} /$ día $( \pm 3,14)(p<0,001)$. Respecto al uso de su audífono en las últimas 2 semanas previas a la aplicación de la encuesta, en el grupo no intervenido sólo el $40 \%$ lo utilizaba más de $8 \mathrm{hrs/}$ día, mientras que en el grupo intervenido el $80 \%$

Tabla 1. Regularidad del uso de audífonos en pacientes con hipoacusia usuarios de audífono sometidos a una intervención para mejorar la adherencia a su uso, basado en el International Outcome Inventory for Hearing Aids (IOI-HA)

\begin{tabular}{|l|c|c|}
\hline Regularidad del uso del audífono & $\begin{array}{c}\text { Grupo no Intervenido }(\mathrm{n}=90) \\
(\%)\end{array}$ & $\begin{array}{c}\text { Grupo intervenido }(\mathrm{n}=90) \\
(\%)\end{array}$ \\
\hline Nunca & 2,22 & 0 \\
\hline Rara vez & 42,22 & 2,22 \\
\hline Ocasionalmente & 46,67 & 17,78 \\
\hline Regularmente & 8,89 & 80 \\
\hline
\end{tabular}

Test exacto de Fisher $p<0,0001$.

Tabla 2. Comparación entre grupos de pacientes con hipoacusia usuarios de audífono sometidos a una intervención para mejorar la adherencia a su uso y no sometidos a la intervención.

Datos obtenidos en base al International Outcome Inventory for Hearing Aids (IOI-HA) y a cuestionario de adherencia proporcionado por Bevan Yueh, MD

\begin{tabular}{|l|c|c|c|}
\hline Variable & $\begin{array}{c}\text { Grupo no intervenido } \\
(\mathrm{n}=90)\end{array}$ & $\begin{array}{c}\text { Grupo intervenido } \\
(\mathrm{n}=90)\end{array}$ & p value \\
\hline $\begin{array}{l}\text { Cantidad de días a la } \\
\text { semana }\end{array}$ & $6( \pm 1,7)$ & $6,53( \pm 1,1)$ & 0,03 \\
\hline $\begin{array}{l}\text { Cantidad de horas al } \\
\text { día }\end{array}$ & $6,9( \pm 3,55)$ & $9,6( \pm 3,14)$ & $<0,001$ \\
\hline $\begin{array}{l}\text { Más de 8 horas al día } \\
\text { durante las últimas 2 } \\
\text { semanas }\end{array}$ & $40 \%$ & $80 \%$ & $<0,001$ \\
\hline
\end{tabular}

${ }^{1}$ Promedio ( \pm Desviación estándar)

2 Frecuencia relativa 
lo usaba más de 8 hrs/día ( $p<0,001)$ (Tabla 2). En suma, al comparar los grupos se obtuvo diferencias significativas en cuanto a la regularidad del uso del audífono, la cantidad de días a la semana que lo utilizaban, la cantidad de horas al día y las horas que utilizaron el audífono durante las últimas dos semanas.

\section{DISCUSIÓN}

La evidencia actual sugiere que las intervenciones de rehabilitación, tanto individuales como grupales, generan resultados positivos ${ }^{24,28-30}$, sin embargo, su disponibilidad en nuestro medio no es la ideal. Para mejorar esta situación, se han propuesto cambios en el manejo de los pacientes con hipoacusia, mencionándose que la disponibilidad de la gama de intervenciones de rehabilitación debe ser mejorada, y los adultos mayores con discapacidad auditiva deben ser invitados a participar activamente en su rehabilitación $n^{31}$.

En el presente estudio se observa que existen diferencias significativas respecto al tiempo de uso y adherencia al uso de audífonos al utilizar esta estrategia de consejería y educación basada en resolución de problemas. En los pacientes beneficiarios sometidos a la intervención, se logró optimizar un programa de alta importancia social, educacional y de rehabilitación, generando un beneficio directo para los usuarios, a través de un programa estructurado de acuerdo a las necesidades particulares de cada grupo de pacientes intervenidos. Es importante destacar que en la presente intervención los grupos fueron comparables entre sí, con una adecuada asistencia de los pacientes intervenidos a las sesiones de rehabilitación, pudiéndose contactar a todos los pacientes enrolados en la evaluación a los 6 meses.

Algunos aspectos que se deben considerar al analizar estos datos es que, en primer lugar, no existe consenso internacional respecto de qué se considera una adecuada adherencia al uso de audífonos. Además, es difícil encontrar instrumentos que midan esto. Por este motivo, para el presente estudio se decidió evaluar la adherencia con una de las preguntas del IOI-HA y con la batería de preguntas que un autor reconocido en la materia utiliza ${ }^{13}$. Sin embargo, sería mejor si existiera un método más objetivo para evaluar esto, lo que haría que los estudios fueran más comparables entre sí. Por último, estos resultados muestran que incluso los pacientes que no fueron sometidos a la intervención presentaban cifras de adherencia y uso del audífono considerablemente buenas: 6 días a la semana, y 6,9 horas al día en promedio. La mayor diferencia se aprecia con la pregunta que hace referencia a la regularidad del uso, lo que podría traducir que quienes fueron sometidos al programa de rehabilitación, a pesar de que efectivamente parecen usar más el audífono, podrían tener una mayor percepción de beneficio del uso de éste. Este aspecto queda pendiente para ser evaluado en futuras investigaciones.

En un estudio clínico realizado recientemente se evaluó si existían diferencias en la intervención con visitas periódicas para aumentar la adherencia entre intervenciones grupales 0 individuales, observándose que no había diferencias significativas respecto a los resultados en logros auditivos, la adherencia al audífono, o las visitas no planificadas ${ }^{31}$. Esto respalda el concepto de que el uso de intervenciones grupales puede mantener una atención de calidad y permitiría destinar horas clínicas hacia otras actividades relevantes como el suministro de audífonos a un mayor número de pacientes, lo que reduciría los tiempos de espera, o el pasar más tiempo con los pacientes que tienen necesidades de rehabilitación más complejas ${ }^{26}$. En este sentido, esto respalda la presente intervención, por lo que se plantea como una intervención útil y efectiva. Utilizar una variedad de herramientas educativas para aumentar las oportunidades de aprendizaje de los pacientes, permitiría una mejor efectividad clínica de las sesiones. Al mejorar la adherencia terapéutica al uso de audífonos, se puede obtener un impacto directo en términos de mejoría de calidad de vida de los usuarios, e indirecto en términos de mejor utilización de un recurso que tiene un costo anual elevado para el país. La intervención implementada impresiona tener buenos resultados preliminares en términos de tiempo de uso del audífono, por lo que sería de utilidad implementarla como parte de la política GES y difundir su uso.

\section{CONCLUSIÓN}

El programa de rehabilitación auditiva Active Communication Education impresiona ser eficaz para 
mejorar la adherencia al uso del audífono en pacientes adultos mayores con hipoacusia, por lo que podría ser una herramienta útil en el manejo de estos pacientes. Por esta razón, estos resultados sugieren que la incorporación de programas de rehabilitación auditiva en pacientes usuarios de audífonos es beneficiosa por lo beneficios directos e indirectos que podría producir.

\section{Agradecimientos}

Los autores desean agradecer a la Sociedad Chilena de Otorrinolaringología, Medicina y Cirugía de Cabeza y Cuello por al apoyo para la realización del presente estudio, y al Dr. Bevan Yueh por facilitar los instrumentos para evaluar adherencia.

Trabajo financiado con fondos del Concurso Anual de Investigación de la Sociedad Chilena de Otorrinolaringología y con fondos del VIII Concurso Nacional de Proyectos de Investigación en Salud (Fonis) SA11l2199.

\section{REFERENCIAS}

1. Yueh B, Shapiro N, Maclean CH, Shekelle PG. Screening and management of adult hearing loss in primary care: scientific review. JAMA 2003; 289(15): 1976-85.

2. Van Eyken E, Van Camp G, Van Laer L. The complexity of age-related hearing impairment: contributing environmental and genetic factors. Audio Neurootol 2007; 12(6): 345-58.

3. Gates Ga, Cooper JC JR., Kannel WB, Miller NJ. Hearing in the elderly: the Framinghan cohort, 1983-1985. Part 1. Basic audiometric tests results. Ear Hear 1990; 11(4): 257-256.

4. Guía Clínica para la Atención Primaria de las Personas Adultas Mayores OPS/OMS-2003.

5. Ministerio de Salud, Chile, Encuesta Nacional de Salud año 2003. http://epi.minsal.cl/epi/html/ invest/ENS/folletoENS.pdf (Revisión actualizada el 24 de agosto del año 2010).

6. Gates GA, Cobb JL, Linn RT, Rees T, Wolf PA, D'Agostino RB. Central auditory dysfunction, cognitive dysfunction, and dementia in older people. Arch Otolaryngol Head Neck Surg 1996; 122(2): 161-7.

7. Guía Clínica Hipoacusia bilateral en personas de 65 años y más que requieren uso de audífono. http://www.redsalud.gov.cl/archivos/guiasges/ hipoacusia.pdf (Revisión actualizada el 24 de agosto del año 2010).

8. Nachtegaal J, Smit J, Smits C, Bezemer P, Van Beek J, Festen J, et al. The Association Between Hearing Status and Psychosocial Health Before the Age of 70 Years: Results From an InternetBased National Survey on Hearing. Ear \& Hearing 2009; 30: 302-12.

9. Hallam R, Ashton P, Sherbourne K, Gailey L. Acquired profound hearing loss: mental health and other characteristics of a large sample. Int J Audiol 2006; 45(12): 715-23.

10. Mahapatra S. Psychiatric and Psychosomatic Illnes in the Deaf. Brit J Psychiat 1974; 125: 450-1.

11. Hearing loss: A growing problem that affects quality of life. National Academy on an Aging Society. Number 2, December 1999.

12. Chisolm T, Abrams A, McArdle J. Short- and long- term outcomes of adult audiological rehabilitation. Ear and Hearing 2004; 25: 5.

13. Yueh B, Souza PE, McDowell JA, et al. Randomized trial of amplification strategies. Arch Otolaryngol Head Neck Surg 2001; 127(10): 1197-1204.

14. Popelka MM, Cruickshanks KJ, Wiley TL, Tweed TS, KLEIN BE, KLEIN R. Low prevalence of hearing aid use among older adults with hearing loss: the Epidemiology of Hearing Loss Study. J Am Geriatr Soc 1998; 46 (9): 1075-8.

15. Mulrow CD, Aguilar C, Endicott JE, et al. Quality-oflife changes and hearing impairment. A randomized trial. Ann Intern Med 1990; 113 (3): 188-94.

16. Dunlop RJ, Beck LB, Dennis KC, Gonzenbach SA, Abrams hB, Berardino JT, Styer SA, Hall A. Support personnel in VA audiology. Audiology Today 2006; 18(1): 24-5.

17. Svard I, Spens KE, Back L, Ahlner BH, Barrenas $M L$. The benefit method: fitting hearing aids in noise. Noise Health 2005; 7(29): 12-23.

18. GarSTeCKI DC, ERLER SF. Hearing loss, control, and demographic factors influencing hearing aid use among older adults. J Speech Lang Hear Res 1998; 41(3): 527-37.

19. Food and Drug Administrations Regulations: Hearing aid devices: professional and patient labeling and condition or sale. Audiology Update 1993; 12: 3-5.

20. Bertoli S, Staehelin K, Zemp E, Schindler C, Bodmer $D$, Probst R. Survey on hearing aid use and 
satisfaction in Switzerland and their determinants. Int J Audiol 2009; 48(4): 183-95.

21. Gussekloo J, de Bont LE, von Faber M, et al. Auditory rehabilitation of older people from the general population-the Leiden 85-plus study. Br J Gen Pract 2003; 53(492): 536-40.

22. KochKIN S, MarkeTrak IV. What is the viable market for hearing aids? Hearing J 1997; 50 (1): 31-9.

23. PacAL JT, YUeH B. Hearing deficits in the older patient: "I didn't notice anything". JAMA 2012; 307(11): 1185-94.

24. Laplante-Lévesque A, Hickson L, Worrall L. Rehabilitation of older adults with hearing impairment: a critical review. J Aging Health 2010; 22(2): 143-53.

25. Hickson L, Worrall L, Scarinci N. A randomized controlled trial evaluating the active communication education program for older people with hearing impairment. Ear Hear 2007; 28(2): 212-30.

26. Sabate E. WHO. Adherence Meeting Report. Genove. World Health Organization. 2001.
27. HAYneS RB. Introduction. En: Haynes RB, Taylor DW, Sackett DI. Editors. Compliance in health care. Baltimore: Johns Hopkins University Press. 1979.

28. Sweetow R, Palmer CV. Efficacy of individual auditory training in adults: A systematic review of the evidence. J Am Acad Audiol 2005; 16(7): 494-504.

29. HawkIns DB. Effectiveness of counselling-based adult group aural rehabilitation programs: $A$ systematic review of the evidence. J Am Acad Audiol 2005; 16(7): 485-93.

30. Chisolm T, Arnold M. Evidence about the effectiveness of aural rehabilitation programs for adults. 2012. En: L. Wong y L. Hickson. Evidence-based practice in Audiology. Evaluating interventions for children and adults with hearing impairment. Plural Publishing: San Diego.

31. Collins M, Liu C, Taylor L, Souza PE, Yueh B. Hearing aid effectiveness after aural rehabilitation: Individual versus group trial results. JRRD 2013; 50(4): 585-98. 